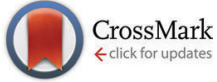

Cite this: Phys. Chem. Chem. Phys., 2015, 17, 20178

Received 10th June 2015, Accepted 10th July 2015 DOI: $10.1039 / c 5 c p 03370 e$

www.rsc.org/pccp

\section{Deformation twinning and the role of amino acids and magnesium in calcite hardness from molecular simulation}

\author{
A. S. Côté, * R. Darkins and D. M. Duffy
}

We employ classical molecular dynamics to calculate elastic properties and to model the nucleation and propagation of deformation twins in calcite, both as a pure crystal and with magnesium and aspartate inclusions. The twinning is induced by applying uniaxial strain to the crystal and relaxing all stress components except the uniaxial component. A detailed analysis of the atomistic processes reveal that the twinning mechanism involves small displacements of the $\mathrm{Ca}$ ions and cooperative rotations of the $\mathrm{CO}_{3}$ ions. The volume of the twinned region expands under increased uniaxial strain via the propagation of steps along the twin boundaries. The energy cost of the twin boundaries is compensated by the reduced hydrostatic stress and strain energy. The presence of biogenic impurities is shown to decrease the strain required to induce twin formation in calcite and, thus, the yield stress. This increased propensity for twinning provides a possible explanation for the increased hardness and penetration resistance observed experimentally in biominerals.

\section{Introduction}

Biominerals produced by living organisms exhibit remarkable mechanical properties, rivalling those of engineered ceramics at high temperatures and pressures. ${ }^{1-3}$ Understanding the mechanisms involved in the superior mechanical properties of biominerals is essential for the design of new materials that reproduce these properties, and has been the aim of several studies over the past few decades. ${ }^{4-7}$ One of the most widely studied biominerals is calcite, the most stable polymorph of $\mathrm{CaCO}_{3}$. Apart from being a rock-forming mineral abundant in the Earth's crust, calcite is also biogenic. It often forms part of the skeleton or exoskeleton of invertebrates, where exceptional hardness and toughness are required for protection and support. These mechanical properties are strongly influenced by a range of additives, including inorganic ions and organic macromolecules. ${ }^{8-11}$ These composite structures can effectively distribute stress and control crack propagation through the material, achieving the required toughness and penetration resistance. ${ }^{12-14}$ One way that this is achieved is deformation twinning, as was recently shown using nanoindentation on a biomineralised calcite exoskeleton. $^{15}$

Deformation twinning is a mechanism which occurs in some crystals when they are subjected to a sufficiently large shear stress. Crystalline twins are characterised by the formation of a mirror

Department of Physics \& Astronomy, University College London, Gower Street, London WC1E 6BT, UK. E-mail: a.cote@ucl.ac.uk plane which separates the original "parent" and "twinned" regions. The highly co-ordinated atomic movement that leads to the twin boundary formation has been extensively studied for metals and minerals. ${ }^{16,17}$ Twinning has a very important role in a range of physical properties of crystals, as well as growth and phase transformations. It has been shown that, at the nanoscale, twin boundaries increase hardness in the same way as grain boundaries ${ }^{18,19}$ by inhibiting dislocation motion, while gliding of dislocations along densely distributed twin boundaries increases fracture toughness. This was recently exemplified by the creation of nanotwinned diamond with exceptional hardness and thermal stability. ${ }^{20}$

Most of the computational simulations of twinning have focused on metals; to our knowledge there have been no previous studies that follow the formation and growth of twins in minerals, but only simulations that analyse pre-formed twins. ${ }^{21-24}$ In this study we examine twinning in calcite using molecular dynamics. We analyse the formation and development of the twin boundaries that are created by the application of a uniaxial strain and calculate the resulting stress-strain curve. We repeat the simulations in the presence of biogenic impurities known to affect mechanical properties ( $\mathrm{Mg}$ ions and aspartate), ${ }^{25-27}$ in order to investigate the superior mechanical properties of biogenic calcite.

\section{Twinning in calcite}

There are four well-identified twin planes in calcite crystals, $e=\{018\}, r=\{104\}, f=\{012\}$ and $c=\{001\}$, introduced either by 


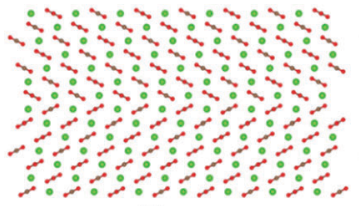

(a)

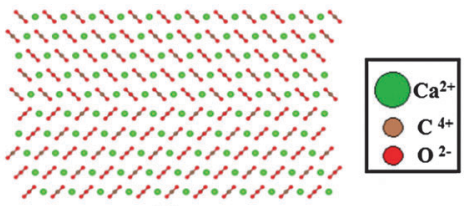

(b)
Fig. 1 (a) $e=\{018\}$ and (b) $r=\{104\}$ twins viewed along the $\langle 010\rangle$ direction.

deformation or as defects during crystal growth. $e$ and $r$ are the most common deformation twinning planes, depicted in Fig. 1. It should also be noted that the $r$-plane is the usual slip plane. The $e$-twin is the one most commonly observed experimentally, and it can be easily introduced by indentation. ${ }^{15,28}$

Since the late nineteenth century there have been numerous studies on naturally and experimentally deformed calcite, confirming the above twin planes. ${ }^{29-32}$ Other carbonate minerals, such as dolomite $\mathrm{CaMg}\left(\mathrm{CO}_{3}\right)_{2}$, have also been analysed in terms of their twin structure and necessary shearing directions to produce twins. ${ }^{33,34}$ However, the atomistic mechanism of nucleation and propagation of twin boundaries in calcite has not been studied computationally and this is one of the aims of the current study.

\section{Computational method}

Molecular dynamics calculations were performed in the DL_POLY Classic code (v. 1.9), ${ }^{35}$ using a rigid ion version of the interatomic potential by Pavese et $a l^{36,37}$ This potential was found to reproduce the mechanical properties of calcite better than the more recent potential by Raiteri et al., ${ }^{38,39}$ which is unsurprising since it was specifically fitted to elastic and vibrational data.

The periodic supercell used in the simulations was a $7 \times 4 \times 3$ multiplication of the conventional orthorhombic cell of calcite, containing $1008 \mathrm{CaCO}_{3}$ units, and with the $z$-axis corresponding to the $c$-axis of the crystal. The electrostatics were handled by the Ewald summation method and the short-range potentials had a cutoff of $10.1 \AA$ A. The simulation timestep was $1 \mathrm{fs}$, using the Nosé-Hoover thermostat at $300 \mathrm{~K}$ with a $100 \mathrm{fs}$ relaxation time. The initial system was relaxed to equilibrium using the $N \sigma T$ ensemble with a $1 \mathrm{ps}$ barostat, for a duration of $1 \mathrm{~ns}$.

Small concentrations of impurities were added to the supercell to examine their effect on the elasticity and deformation twinning. We added 5 and $10 \mathrm{~mol} \% \mathrm{Mg}$ in our supercell by random substitution of $\mathrm{Ca}^{2+}$ ions, and modelled the $\mathrm{Mg}$ interactions with the potential by de Leeuw for $\mathrm{MgCO}_{3} \cdot{ }^{40}$ We repeated the simulations with 0.4 and $0.8 \mathrm{~mol} \%$ aspartate (Asp). The zwitterionic form of aspartate $\left(\mathrm{Asp}^{1-}\right)$ was used $\left({ }^{+} \mathrm{H}_{3} \mathrm{~N}-\mathrm{CH}-\left(\mathrm{CH}_{2} \mathrm{CO}_{2}{ }^{-}\right)-\mathrm{CO}_{2}{ }^{-}\right)$, and its potential was generated with AMBER. ${ }^{41}$ The interactions between aspartate and calcite were fitted using the methods described in ref. 42. The configuration, in this case, was obtained by using metadynamics to drive the crystallisation of disordered calcium carbonate that contained the aspartate molecules. The resulting structure is shown in Fig. 2 where it can be seen that the amino group in the aspartate takes the place of a $\mathrm{Ca}$ ion in the calcite lattice,
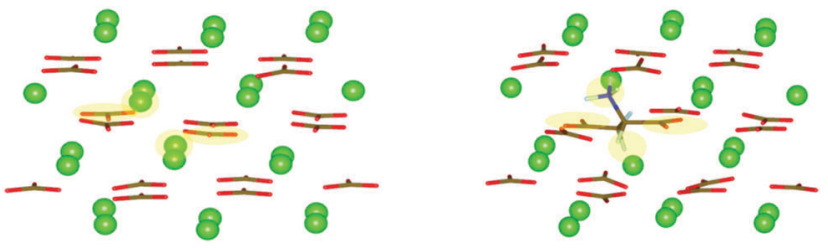

Fig. 2 Snapshots before (left) and after (right) the inclusion of Asp in the calcite lattice. The positions of the substituted ions are shaded.

while the two carboxylic acid groups replace two carbonate groups, hence the aspartate fits in the calcite lattice with minimal disruption.

\subsection{Elastic properties}

We used the small strain method to calculate the six independent elastic constants $\left(C_{11}, C_{33}, C_{44}, C_{12}, C_{13}, C_{14}\right)$ of calcite, with and without $\mathrm{Mg}$ and Asp impurities. Small (up to $\pm 1 \%$ ) tensile, compressive and shear strains were applied to the equilibrium lattice vectors, and the resulting stresses calculated. ${ }^{43}$ This was repeated for 20 strain increments close to the equilibrium vectors, with each strain direction corresponding to one or more elastic constants. Specifically, given an initial orthorhombic calcite supercell with axes $a, b$ and $c$, the three matrices chosen to define the axes of the deformed supercell were the following, with an increment $\delta=0.001 \AA$ :

$$
\left(\begin{array}{ccc}
(1+\delta) a & 0 & 0 \\
0 & b & 0 \\
0 & 0 & c
\end{array}\right),\left(\begin{array}{ccc}
a & 0 & 0 \\
0 & b & 0 \\
0 & 0 & (1+\delta) c
\end{array}\right) \text {, and }\left(\begin{array}{ccc}
a & \delta a & 0 \\
\delta b & b & 0 \\
0 & 0 & c
\end{array}\right)
$$

from which all the elastic constants were obtained.

The elastic constants were extracted by a linear fit to the stress-strain data $\left(\sigma_{i}=C_{i j} \varepsilon_{j}\right)$. This is possible because the strain is sufficiently small that the deformation stays in the linear regime. The stresses were calculated by sampling the canonical ensemble for $0.2 \mathrm{~ns}$ after each strain increment. The bulk and shear moduli were calculated from the elastic constants using the Voigt-Reuss-Hill averaging scheme. ${ }^{44}$

\subsection{Deformation twinning}

A similar method was used to deform the supercell along the $z$-direction, i.e. the $c$-axis of the unstrained and relaxed unit cell. The implementation of the $N \sigma T$ ensemble in DL_POLY was modified in order to constrain the deformation in the $z$ direction only. All other components of the cell vectors were relaxed to relieve all stress components except $\sigma_{z}$. Thus, in general, all components of the strain vector are finite and all components of the stress vector are zero except $\sigma_{z}$. We are effectively simulating an experimental setup in which only $\varepsilon_{z}$ is constrained.

The supercell, including the atomic coordinates, was stretched in small incremental steps at a rate of $0.15 \mathrm{~m} \mathrm{~s}^{-1}$, up to $\sim 30 \%$ strain. The strain increase at each step was $0.28 \%$ and a $100 \mathrm{ps}$ constant strain simulation at $300 \mathrm{~K}$ was carried out after each step. The final configurations of these 100 ps simulations were stretched and used as the starting configuration for the 
next step. The $z$-component of the stress - the only non-zero component - was calculated after each strain increment by averaging over the final 98 ps of the 100 ps constant strain simulations. The calculated values were used to plot the uniaxial stress-strain curve. The atomistic configuration was also captured after each strain increment and the atomic motion involved in twin formation and growth was studied in detail.

\subsection{Calculation of local stress field}

In addition to calculating the average uniaxial stress for the simulation cell, we calculated the local hydrostatic stress distribution throughout the cell. This gives information about the stress distribution near the twin boundaries and the stress introduced by the impurities. We also use this to compare the stress distribution in the simulation cell before and after twinning.

A method for calculating stress fields in ionic crystals from molecular dynamics was introduced by Branicio and Srolovitz ${ }^{45}$ and applied by Darkins et al. $^{46}$ The continuous stress field is derived from the time-averaged atomic virials, $\left\langle W_{i^{\alpha} \beta}\right\rangle$, using

$$
\Pi^{\alpha \beta}(r)=\sum_{i \in X} P\left(r-r_{i}\right) W_{i^{\alpha \beta}}
$$

where all species $X$ and atoms $i$ are summed, and $P(r)$ is a threedimensional normalised Gaussian of the form

$$
P(r)=\frac{27}{R^{3}(2 \pi)^{3 / 2}} \exp \left(-9 r^{2} / 2 R^{2}\right),
$$

where $R$ parametrises the size of the Gaussian and is equivalent to three standard deviations. In our calculations $R$ was set to $4 \AA$ A. $\alpha$ and $\beta$ take on values $x, y, z$ to generate the components of the virial stress tensor. The supercell is divided into a grid, and the hydrostatic stress field $\operatorname{Tr}(\Pi(r)) / 3$, which is a scalar field equivalent to the negative of the pressure field, is calculated at every grid point using eqn (1). By using a dense grid of $1 \AA$ divisions, we can examine the stress distribution throughout our system, both before and after deformation. The calculations were performed at a very low temperature $(10 \mathrm{~K})$ to reduce thermal noise.

\section{Results and discussion}

\subsection{Elastic properties}

The calculated elastic constants of calcite, with and without $\mathrm{Mg}$ and Asp impurities, are presented in Table 1. The calculated values are in good agreement with both the values calculated previously with a different methodology ${ }^{37}$ and the measured values. These values give us confidence that the potentials we use are appropriate for modelling the mechanical properties of calcite.

The addition of $\mathrm{Mg}$ makes calcite stiffer - the $10 \mathrm{~mol} \%$ impurity caused a $2 \%$ increase in $C_{11}$. This increase in stiffness is in agreement with studies on dolomite, which has considerably higher elastic moduli compared to calcite. ${ }^{47}$ Asp has the opposite but larger effect, achieving approximately the same change by including only $0.8 \mathrm{~mol} \%$ of impurity. As the concentrations used here were small, the symmetry of the elastic matrix remained unaffected.

\subsection{Deformation twinning}

The calculated stress-strain curve for pure calcite up to a uniaxial strain of $25 \%$ is shown in Fig. 3. The curve is linear up to $2 \%$ strain and the gradient gives a tensile modulus of $66.4 \pm 0.2 \mathrm{GPa}$, which is in excellent agreement with the value calculated from the elastic constants in section 4.1 above. The curve is close to linear up to a strain of $10.8 \%$, at which point there is a sharp drop in stress, signalling the formation of a pair of $\{104\}$ twin boundaries separated by 2-3 atomic layers.

The stress at which the stress-strain curve deviates significantly from linear behaviour, the yield stress, was found to be 7.7 GPa. We note that the calculated yield stress and strain are much higher than would be achieved in a real calcite crystal since, in our model, there are no surfaces, cracks or other flaws that would usually serve as nucleation sites for such failure.

The atomistic configuration of the simulation cell immediately after the formation of the pair of deformation twins is shown in the second snapshot of Fig. 3. The twinned volume between the two twins is two or three atomic layers thick and has a different crystallographic orientation to the original (parent) crystal. The change in the number of layers is the result of "steps" in each of the twin boundaries. There can be one or more steps in each twin, and their number increases with strain. We repeated this experiment using a simulation cell that was 1.5 times smaller in each direction, and we found that $r$-twins formed at the same stress/strain values as in the original cell; the only difference was that the twin boundaries spanned the entire cell, contrary to the ones shown in Fig. 3, which end part way through.

\subsection{Twin propagation}

The stages of deformation were identified by analysing the trajectories of each simulation. The motion of the twin boundary steps results in widening of the twinned region, with increased

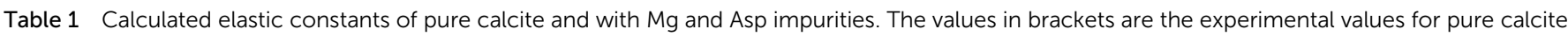

\begin{tabular}{|c|c|c|c|c|c|c|c|c|c|}
\hline & $C_{11}$ & $C_{33}$ & $C_{44}$ & $C_{12}$ & $C_{13}$ & $C_{14}$ & $K_{\mathrm{VRH}}$ & $G_{\mathrm{VRH}}$ & $Y_{\mathrm{zz}}$ \\
\hline Pure calcite & $156.6(149.4)$ & $92.1(85.2)$ & $35.1(34.1)$ & $57.9(57.9)$ & $52.6(53.5)$ & $-12.2(-20.0)$ & $78.4(76.1)$ & $37.6(32.8)$ & $66.3(57.6)$ \\
\hline$+5 \% \mathrm{Mg}$ & 157.7 & 92.2 & 35.2 & 58.1 & 52.3 & -11.6 & 78.5 & 38.2 & 66.8 \\
\hline$+10 \% \mathrm{Mg}$ & 159.8 & 93.6 & 35.2 & 59.3 & 52.7 & -11.3 & 79.5 & 38.6 & 68.2 \\
\hline$+0.4 \%$ Asp & 155.1 & 90.6 & 34.3 & 56.9 & 52.3 & -12.5 & 77.5 & 36.9 & 64.8 \\
\hline$+0.8 \%$ Asp & 152.7 & 89.4 & 33.6 & 55.9 & 52.0 & -12.4 & 76.6 & 36.2 & 63.5 \\
\hline
\end{tabular}
from Chen et al. ${ }^{47}$ 


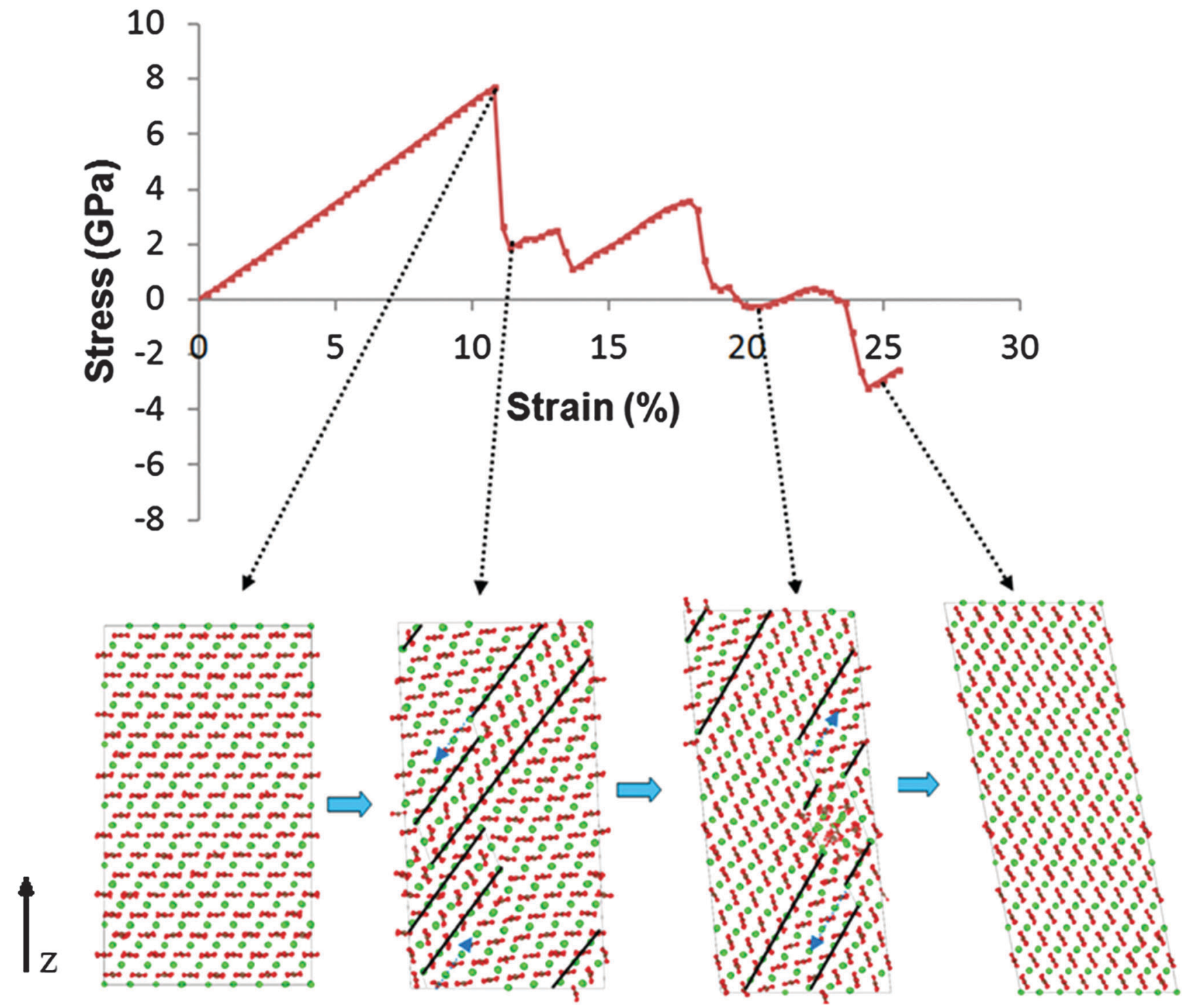

Fig. 3 Deformation twinning and widening of the twinned region caused by tensile strain along the $z$-axis of the calcite supercell. The stress-strain relationship and four configurations are shown between $11 \%$ and $25 \%$ strain. The final configuration has entirely adopted the crystallographic orientation of the twinned region. The dashed blue arrows show the direction of step propagation, which results in widening of the twinned volume. The twin boundaries are drawn as black lines. The thin dashed lines denote the end of the twinned region, as well as the steps in the boundaries.

tensile strain. As the lattice is strained further, the steps move along the boundary, making the twinned region wider with respect to the parent region. When the step reaches the end of the twin boundary, a step nucleates on the opposite side of the twinned region and continues to propagate, further widening the twin region. Steps may also form spontaneously at the end of the twin boundaries. When two steps meet, the two twinned areas join, and in this instance, due to the periodic boundary conditions, the "parent" region is finally consumed and the entire structure adopts the crystallographic orientation of the "twin" region. In the pure calcite lattice this happens at $\sim 25 \%$ strain. The $\alpha$ angle of the supercell also gradually changes to accommodate the new periodic structure, from $90^{\circ}$ to $101^{\circ}$. Snapshots of the process are illustrated in Fig. 3, together with the corresponding stress-strain relationship. Whenever the steps move a large distance or annihilate, we notice a corresponding drop in stress. Interestingly, near $25 \%$ strain, when the parent regions disappear completely, the structure is in a compressed state (negative stress). The energy cost of the twin boundaries is larger than that of a twin-free lattice with stored elastic energy. Specifically, using the initial unstrained lattice as reference, the energy per unit volume drops from $237 \mathrm{MJ} \mathrm{m}^{-3}$ to $64 \mathrm{MJ} \mathrm{m}^{-3}$, before and after the disappearance of the twins.

We repeated the simulations with low concentrations of $\mathrm{Mg}$ (5 mol\%) and Asp (0.4 mol\%) impurities. The twin boundaries that formed following the initial drop in stress are shown in Fig. 4.

In the $\mathrm{Mg}$ case, the twin boundaries started forming in the region of the supercell where the $\mathrm{Mg}$ concentration was higher than the cell average, around $9 \%$. Similarly, in the simulation with Asp impurities, the twinned region initially formed close 


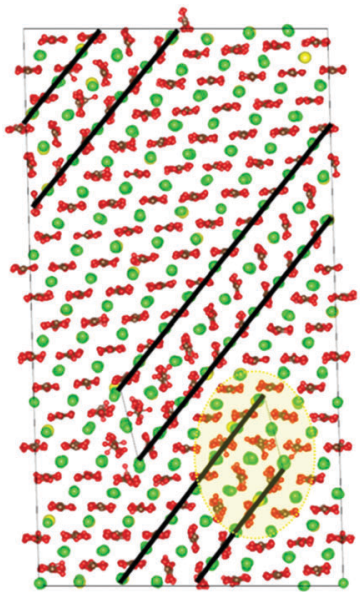

(a)

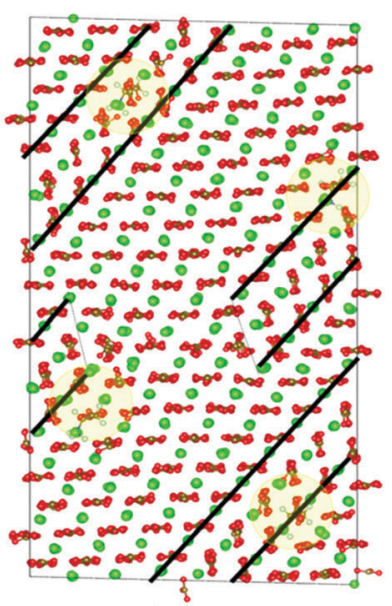

(b)
Fig. 4 (a) $\{104\}$ twins in calcite with 5 mol\% Mg. The shaded regions shows the area with the largest $\mathrm{Mg}^{2+}$ concentration. The twin nucleates from that area. $\mathrm{Mg}^{2+}$ ions are coloured yellow. (b) $\{104\}$ twins with $\mathrm{Asp}^{1-}$; here the shaded circles mark the position of the Asp ${ }^{1-}$ molecules.

to the amino acid molecules. We attribute this to high localised stress fields around the impurities and we discuss this further in Section 4.4.

The calculated stress-strain curves for calcite with $\mathrm{Mg}$ and Asp impurities are shown in Fig. 5. The curve for pure calcite is included for comparison. We note that the initial twin formation occurs at lower strain (9.7\% for $\mathrm{Mg}, 9.4 \%$ for Asp) and stress (6.7 GPa for $\mathrm{Mg}$, 6.1 GPa for Asp) values in the presence of impurities. This is an important result as it implies that impurities enhance twin boundary formation and, consequently, reduce the yield stress of calcite.

\subsection{Stress field}

We calculated the hydrostatic stress fields in our supercells with both $\mathrm{Mg}$ and Asp impurity ions. A volumetric representation of the stress field in the $5 \mathrm{~mol} \% \mathrm{Mg}$ supercell, just before and just after the twin boundaries form, are shown in Fig. 6 .

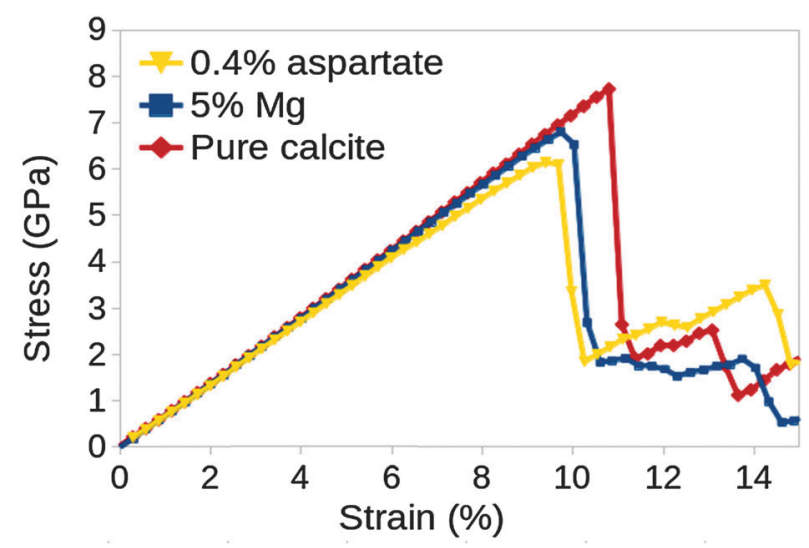

Fig. 5 Comparison of the stress-strain relationship between the system with $5 \% \mathrm{Mg}, 0.4 \% \mathrm{Asp}$, and the pure calcite system. Twinning occurs at lower stress when impurities are introduced in the calcite lattice.

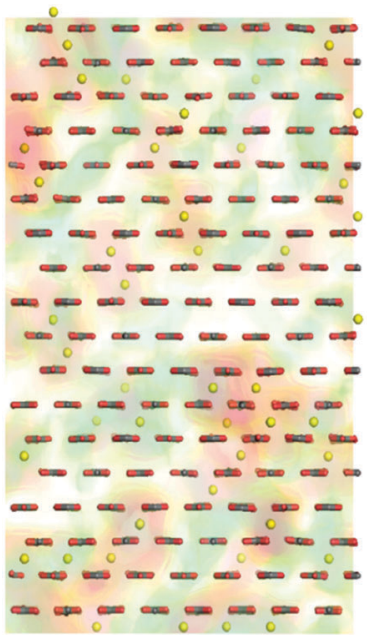

(a)

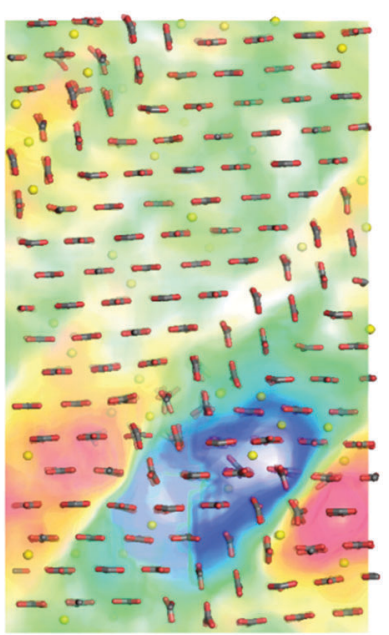

(b)

\section{$-2.5 \mathrm{GPa}$}

$2.5 \mathrm{GPa}$

Fig. 6 Volume rendering of hydrostatic stress (a) before and (b) after deformation twinning. Calcium ions are not shown for clarity; magnesium ions are shown in yellow. The deformed structure on the right corresponds to Fig. 4(a).

As expected, the volume with the highest local stress before deformation is the volume with the largest impurity concentration.

The $\mathrm{Mg}^{2+}$ ions, being smaller than the $\mathrm{Ca}^{2+}$ ions they substituted, cause a compressive stress around them in the lattice. When there is a high localised impurity concentration, the stress in that volume is higher and the deformation twin nucleates close to that region in order to relieve the stress. The compressive (positive) stress around the $\mathrm{Mg}^{2+}$ ions at the moment just before twinning is visible in Fig. 6. After the twin has formed, the previously-compressed region is now replaced by a pocket of negative stress. The same effect was observed when Asp was the impurity; the twins form near the impurities and act to relieve the local accumulation of stress.

\section{Conclusions}

We have used molecular dynamics to calculate the stress-strain curve for calcite and to observe the process of deformation twinning induced by uniaxial strain in the (001) direction. We observe elastic deformation up to a strain of around $10 \%$. We note that this is much higher than would be expected in an experimental sample where grain boundaries and other defects introduce stress concentrations that initiate failure. Our simulations are instead representative of a perfect calcite crystal and provide detailed information about intrinsic deformation mechanisms.

At a strain of $10.8 \%$, and a stress of $7.7 \mathrm{GPa}$, a pair of $\{104\}$ twin boundaries form by small displacements of the $\mathrm{Ca}$ ions and displacement and cooperative rotation of the $\mathrm{CO}_{3}$ ions. The twin boundaries are initially separated by two to three crystal layers with a different orientation to the original crystal, with steps in the twin boundaries separating the two- and three-layer regions. As the strain is increased further, the volume of the 
twinned region expands by propagation of the steps along the boundaries, further reducing the uniaxial stress.

We repeated the simulations with $\mathrm{Mg}$ and aspartate impurities, which are additives commonly found in biogenic calcite. The calculated stress-strain curves for the impure calcite crystals were similar to that for pure calcite. However, the initial drop in stress, corresponding to the formation of the pair of twin boundaries, occurred at significantly lower strains and stresses $(9.7 \%$ and $6.7 \mathrm{GPa}$ for $\mathrm{Mg}$ and $9.4 \%$ and $6.1 \mathrm{GPa}$ for Asp). The twin boundaries formed initially close to the regions with the highest impurity concentration and analysis of the local stress distribution around the impurities before and after twin formation showed that twin formation affects the local stress associated with the defects.

The fact that impurities enhance deformation twinning may explain the enhanced hardness and penetration resistance of biogenic calcite in nanoindentation experiments. ${ }^{15,48}$ The inclusion of impurity ions, amino acids and proteins will result in a higher density of twin boundaries in response to the application of stress. The twin boundaries, in turn, will inhibit dislocation motion and nano-crack propagation, increasing both hardness and penetration resistance. Deformation twinning may therefore prove to be a key mechanism in the effort to engineer biomimetic minerals with superior mechanical properties.

In summary, we have used molecular dynamics to investigate the response of calcite to uniaxial strain, with and without added impurities. We have found that plastic deformation occurs via the formation of pairs of $\{104\}$ twin boundaries and proceeds via the motion of steps along these boundaries. Added impurities reduce the stress and strain required for twin formation, which may contribute to the enhanced hardness of biogenic calcite.

\section{Acknowledgements}

This work was supported by the Engineering and Physical Sciences Research Council (grant no. EP/I001514/1). This programme grant funds the Materials Interface with Biology (MiB) consortium. R.D. acknowledges funding from the EPSRC under the Molecular Modelling and Materials Science Industrial Doctorate Centre and from the Pacific Northwestern National Laboratory.

\section{Notes and references}

1 S. Mann, Biomineralization: the form(id)able part of bioinorganic chemistry!, J. Chem. Soc., Dalton Trans., 1997, 3953-3962.

2 S. Weiner and P. M. Dove, An overview of biomineralization processes and the problem of vital effect, Rev. Mineral. Geochem., 2003, 54, 1-31.

3 E. Beniash, Biominerals-hierarchical nanocomposites: the example of bone, Wiley Interdiscip. Rev.: Nanomed. Nanobiotechnol., 2011, 3, $47-69$.
4 M. Sarikaya, Biomimetics: Materials fabrication through biology, Proc. Natl. Acad. Sci. U. S. A., 1999, 96, 14183-14185.

5 P. Fratzl, Biomimetic materials research: what can we really learn from nature's structural materials? J. R. Soc., Interface, 2007, 4, 637-642.

6 A. K. Nair, F. Libonati, Z. Qin, L. S. Dimasand and M. J. Buehler, Mechanical and interface properties of biominerals: Atomistic to coarse grain modeling, in Biomineralization Sourcebook, ed. L. Gower, CRC Press, 2014, pp. 337-351.

7 O. S. Bello, K. A. Adegoke and R. O. Oyewole, Biomimetic materials in our world: A review, IOSR J. Appl. Chem., 2013, 5, 22-35.

8 J. Aizenberg, S. Weiner and L. Addadi, Coexistence of amorphous and crystalline calcium carbonate in skeletal tissues, Connect. Tissue Res., 2003, 44, 20-25.

9 F. C. Meldrum, Calcium carbonate in biomineralisation and biomimetic chemistry, Int. Mater. Rev., 2003, 48, 187-224.

10 F. C. Meldrum and H. Cölfen, Controlling mineral morphologies and structures in biological and synthetic systems, Chem. Rev., 2008, 108, 4332.

11 N. A. J. M. Sommerdijk and G. de With, Biomimetic $\mathrm{CaCO}_{3}$ mineralization using designer molecules and interfaces, Chem. Rev., 2008, 108, 4499-4550.

12 F. Barthelat, Biomimetics for next generation materials, Philos. Trans. R. Soc., A, 2007, 365, 2907-2919.

13 P. Fratzl and R. Weinkamer, Nature's hierarchical materials, Prog. Mater. Sci., 2007, 52, 1263-1334.

14 C. Li and D. L. Kaplan, Biomimetic composites via molecular scale self-assembly and biomineralization, Curr. Opin. Solid State Mater. Sci., 2003, 7, 265-271.

$15 \mathrm{~L}$. Li and C. Ortiz, Pervasive nanoscale deformation twinning as a catalyst for efficient energy dissipation in a bioceramic armour, Nature, 2014, 13, 1-7.

$16 \mathrm{~J}$. W. Christian and S. Mahajan, Deformation twinning, Progress in Materials, Science, 2000, 39, 1-157.

17 Y. T. Zhu, X. Z. Liao and X. L. Wu, Deformation twinning in nanocrystalline materials, Prog. Mater. Sci., 2012, 57, 1-62.

18 D. Wolf, V. Yamakov, S. R. Phillpot, A. Mukherjee and H. Gleiter, Deformation of nanocrystalline materials by molecular-dynamics simulation: relationship to experiments? Acta Mater., 2005, 53, 1-40.

19 T. Zhu and H. Gao, Plastic deformation mechanism in nanotwinned metals: An insight from molecular dynamics and mechanistic modeling, Scr. Mater., 2012, 66, 843-848.

20 Q. Huang, D. Yu, B. Xu, W. Hu, Y. Ma, Y. Wang, Z. Zhao, B. Wen, J. He, Z. Liu and Y. Tian, Nanotwinned diamond with unprecedented hardness and stability, Nature, 2014, 510, 250-253.

21 M. Bruno, F. R. Massaro, M. Rubbo, M. Prencipe and D. Aquilano, (10.4), (01.8), (01.2), and (00.1) Twin Laws of Calcite $\left(\mathrm{CaCO}_{3}\right)$ : Equilibrium Geometry of the Twin Boundary Interfaces and Twinning Energy, Cryst. Growth Des., 2010, 10, 3102-3109.

22 A. Toru, N. Kohji and I. Tomonori, Effects of Atomic Arrangements on Structural Stability and Electronic 
Structures of Twin Boundary Interfaces in $\mathrm{CaCO}_{3}$ Calcite, J. Phys. Chem. C, 2012, 116, 987-993.

23 D. Aquilano, M. Bruno, M. Rubbo and P. L. F. R. Massaro, Twin Laws and Energy in Monoclinic Hydroxyapatite, Ca5(PO4)3(OH), Cryst. Growth Des., 2015, 15, 411-418.

24 B. Li and K. M. Knowles, Molecular dynamics simulation of albite twinning and pericline twinning in low albite, Modell. Simul. Mater. Sci. Eng., 2013, 21, 05501.

25 A. J. Andersson, F. T. Mackenzie and N. R. Bates, Life on the margin: implications of ocean acidification on Mg-calcite, high latitude and cold-water marine calcifiers, Mar. Ecol.: Prog. Ser., 2008, 22, 4216.

26 S. Borukhin, L. Bloch, T. Radlauer, A. H. Hill, A. N. Fitch and B. Pokroy, Screening the incorporation of amino acids into an inorganic crystalline host: The case of calcite, Adv. Funct. Mater., 2012, 22, 4216.

27 W.-T. Hou and Q.-L. Feng, Morphologies and Growth Model of Biomimetic Fabricated Calcite Crystals Using Amino Acids and Insoluble Matrix Membranes of Mytilus edulis, Cryst. Growth Des., 2006, 6, 1086-1090.

28 K. Chen, M. Kunz, N. Tamura and H.-R. Wenk, Deformation twinning and residual stress in calcite studied with synchrotron polychromatic X-ray microdiffraction, Phys. Chem. Miner., 2011, 38, 491-500.

29 F. J. Turner, Nature and dynamic interpretation of deformation lamellae in calcite of three marbles, Am. J. Sci., 1953, 251, 276-298.

30 M. Burkhard, Calcite twins, their geometry, appearance and significance as stress-strain markers and indicators of tectonic regime: a review, J. Struct. Geol., 1993, 15, 351-368.

31 J. H. P. De Bresser and C. J. Spiers, High temperature deformation of calcite single crystals by $\mathrm{r}+$ and $\mathrm{f}+$ slip, in Deformation Mechanisms, Rheology and Tectonics, Spec. Publ. - Geol. Soc. London, 1990, 54, 285-298.

32 B. Pokroy, M. Kapon, F. Marin, N. Adir and E. Zolotoyabko, Protein-induced, previously unidentified twin form of calcite, Proc. Natl. Acad. Sci. U. S. A., 2007, 104, 7337-7341.

33 W. F. Bradley, J. F. Burst and D. L. Graf, Crystal chemistry and differential thermal effects of dolomite, Am. Mineral., 1953, 38, 207-217.

34 D. J. Barber and H. R. Wenk, Deformation twinning in calcite, dolomite, and other rhombohedral carbonates, Phys. Chem. Miner., 1979, 5, 141-165.

35 I. T. Todorov, W. Smith, K. Trachenko and M. Dove, DL_POLY_3: new dimensions in molecular dynamics simulations via massive parallelism, J. Mater. Chem., 2006, 16, 1911-1918.
36 A. Pavese, M. Catti, G. D. Price and R. Jackson, Interatomic potentials for $\mathrm{CaCO}_{3}$ polymorphs (calcite and aragonite), fitted to elastic and vibrational data, Phys. Chem. Miner., 1992, 19, 80-87.

37 A. Pavese, M. Catti, S. C. Parker and A. Wall, Modelling of the thermal dependence of structural and elastic properties of calcite $\mathrm{CaCO}_{3}$, Phys. Chem. Miner., 1996, 23, 89-93.

38 P. Raiteri, J. D. Gale, D. Quigley and P. M. Rodger, Derivation of an accurate force-field for simulating the growth of calcium carbonate from aqueous solution: a new model for the calcite-water interface, J. Phys. Chem. C, 2010, 114, 5997-6010.

39 P. Raiteri and J. D. Gale, Water Is the Key to Nonclassical Nucleation of Amorphous Calcium Carbonate, J. Am. Chem. Soc., 2010, 132, 17623-17634.

40 N. H. De Leeuw, Molecular dynamics simulations of the growth inhibiting effect of $\mathrm{Fe}^{2+}, \mathrm{Mg}^{2+}, \mathrm{Cd}^{2+}$, and $\mathrm{Sr}^{2+}$ on calcite crystal growth, J. Phys. Chem. B, 2002, 106, 5241-5249.

41 W. D. Cornell, P. Cieplak, C. I. Bayly, I. R. Gould, K. M. J. Merz, D. M. Ferguson, D. C. Spellmeyer, T. Fox, J. W. Caldwell and P. A. Kollman, A Second Generation Force Field for the Simulation of Proteins, Nucleic Acids, and Organic Molecules, J. Am. Chem. Soc., 1995, 117, 5179-5197.

42 C. L. Freeman, J. H. Harding, D. J. Cooke, J. A. Elliott, J. S. Lardge and D. M. Duffy, New Forcefields for Modeling Biomineralization Processes, J. Phys. Chem. C, 2007, 111, 11943-11951.

43 A. M. Walker, The effect of pressure on the elastic properties and seismic anisotropy of diopside and jadeite from atomic scale simulation, Phys. Earth Planet. Inter., 2012, 192, 81-89.

44 R. Hill, The elastic behaviour of a crystalline aggregate, Proc. Phys. Soc., London, Sect. A, 1952, 65, 349-354.

45 P. S. Branicio and D. J. Srolovitz, Local stress calculation in simulations of multicomponent systems, J. Comput. Phys., 2009, 228, 8467-8479.

46 R. Darkins, M. L. Sushko, J. Liu and D. M. Duffy, Stress in titania nanoparticles: an atomistic study, Phys. Chem. Chem. Phys., 2014, 16, 9441-9447.

47 C.-C. Chen, C.-C. Lin, L.-G. Liu, S. V. Sinogeikin and J. D. Bass, Elasticity of single-crystal calcite and rhodochrosite by Brillouin spectroscopy, Am. Mineral., 2001, 86, 1525-1529.

48 Y.-Y. Kim, L. Ribeiro, F. Maillot, O. Ward, S. Eichhorn and F. Meldrum, Bio-Inspired Synthesis and Mechanical Properties of Calcite-Polymer Particle Composites, Adv. Mater., 2010, 22, 2082. 